\title{
An Approach to Digital Archiving of Art Paintings
}

\author{
Shoji Tominaga \\ Department of Engineering Informatics, \\ Osaka Electro-Communication University, \\ Neyagawa, Osaka 572-8530, Japan \\ shoji@tmlab.osakac.ac.jp \\ http://www.osakac.ac.jp/labs/shoji/English/index_e.html
}

\begin{abstract}
This paper describes an approach to digital archives of art paintings by considering the surface properties and the perceptual effect. A multi-band imaging system with six spectral channels is used for observing the painting surfaces. Multiple images of a painting are acquired with different illumination directions. Algorithms are presented for estimating the surface properties of surface normals, surface spectral reflectance, and reflection model parameters. All the estimates are combined for rendering realistic images of the painting under a variety of illumination and viewing conditions. Moreover, a chromatic adaptation transform is described for predicting appearance of the painting under incandescent illumination and producing the full color image on a display device. The feasibility of the method is demonstrated for an oil painting.
\end{abstract}

\section{Introduction}

Digital archiving of art paintings was originally based on making color-calibrated images from the paintings [1], where the surface of a painting was assumed to be a flat plane and was photographed under diffuse light sources. Practical digital archiving of art paintings including oil paintings and water paintings, however, should be based on both shape information for describing surface geometries and spectral information for color reproduction. Moreover, we have to consider human perceptual effects when the images are rendered on a display device.

A direct way to acquire the shape data of object surfaces is to use a laser rangefinder [2]. This device, however, make unavoidable errors in measuring colored paintings that include specularity. Moreover laser beams may be harmful to the painting surface. Without using the 3D range data, the surface shape of a painting can be imitated using a set of surface normal vectors of small facets [3]. Next, it is well known that the spectral reflectance information is more useful than color information [4]-[5]. In fact, an RGB image is device-dependent and valid for only the fixed conditions of illumination and viewing.

Concerning the perceptual effects, we should note the viewing conditions for art paintings. Most art paintings are hung on the wall indoors, which are often illuminated with incandescent lamps. In this situation, we cannot neglect the effect of chromatic adaptation that is the most important color-appearance phenomenon of the human visual system. Colorimetric image rendering pixel-wise is not enough to produce a realistic appearance of art paintings on any display device. 
The present paper describes an approach to digital archives of art paintings by considering the surface properties and the perceptual effect. Figure 1 depicts the flow for the total digital archives of art paintings. We estimate the surface properties, including surface normal, surface spectral reflectance, and reflection model parameters. A painting surface is observed with a multi-band camera system with six spectral channels. Multiple images of the painting are acquired with different illumination directions. All the estimated surface data are combined for image rendering. Moreover, the image rendered in a colorimetric way is transformed with the chromatic adaptation effect. This chromatic adaptation transform is useful for predicting appearance of the paintings under the illumination of an incandescent lamp and producing the full color images on a monitor.

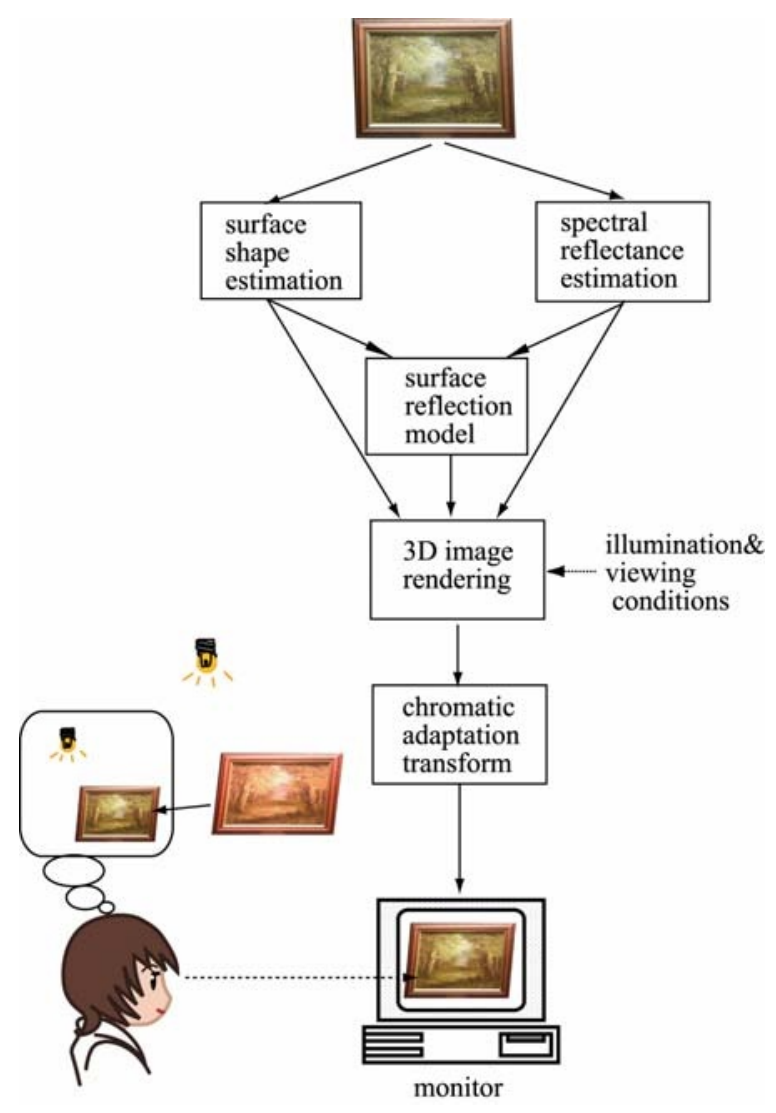

Fig. 1. Flow for the total digital archives of art paintings

\section{Multi-band Measuring System}

Our multi-band measuring system is decomposed into a monochrome camera and a multi-spectral lighting system, as shown in Figure 2. The camera is a CCD camera 
with the image size of 1636x1236 pixels. The bit depth of the image is 10 bits. The lighting system consists of a slide projector and six color filters. Combining the camera and the light source provides a stable imaging system with six-spectral bands in the visible wavelength range of $400-700 \mathrm{~nm}$. The image acquisition of the same object surface is repeated for different illumination directions. The camera aims at the object surface from vertically above the painting. The position of a light source is changed around the optical axis. In practice, multi-band images are captured under nine illumination directions.
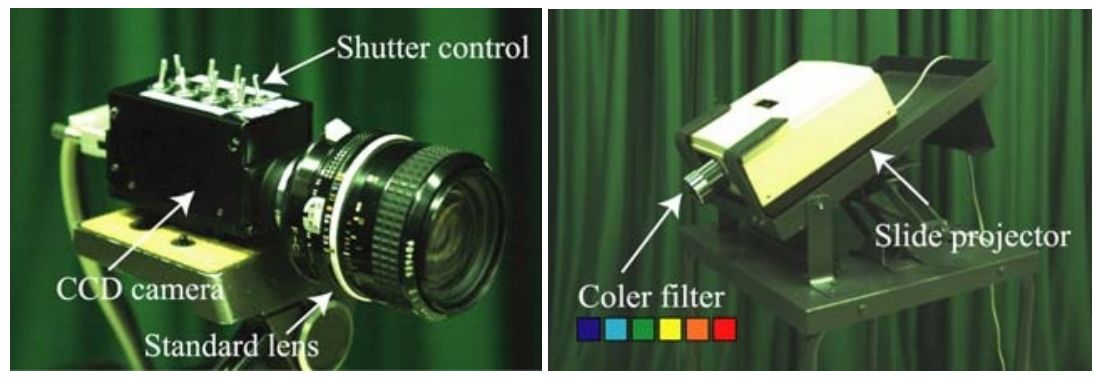

Fig. 2. Multi-band imaging system with six-spectral channels

\section{Modeling of Painting Surfaces}

\subsection{Geometry Model}

\section{(A) 3D Model}

The laser range finder is used for $3 \mathrm{D}$ measurement of the surface shape of a painting. In this system, a horizontal stripe laser beam is emitted to the surface. This light produces a bright spot on the surface that is imaged by a calibrated CCD camera. The $3 \mathrm{D}$ position of the spot is then reconstructed by triangulation. For example, Figure 3 shows the surface shape constructed by regular meshes for a small area of an oil painting. The range data in coordinates $(\mathrm{X}, \mathrm{Y}, \mathrm{Z})$ are depicted in the unit of $\mathrm{mm}$. These 3D measurements by the laser range finder are available for matte surfaces with only diffuse reflection component. However this system makes errors for colored surfaces with gloss and specular highlight.

\section{(B) 2D Model}

The surface of an art painting can be considered as a rough plane rather than a 3D curved surface. Therefore it is not necessary to reconstruct the $3 \mathrm{D}$ surface for digital archiving. An array of surface normal vectors can be used for render the image of a rough plane. The surface normal vector at each pixel point is estimated from a change in shading.

The left figure in Figure 4 illustrates the needle map of the surface normals estimated for a small area of the oil painting. The normal estimation is based on a photometric stereo, which uses the camera data at several illumination directions 
without any specular reflection component. The right figure shows an image rendering the array of the surface normals.

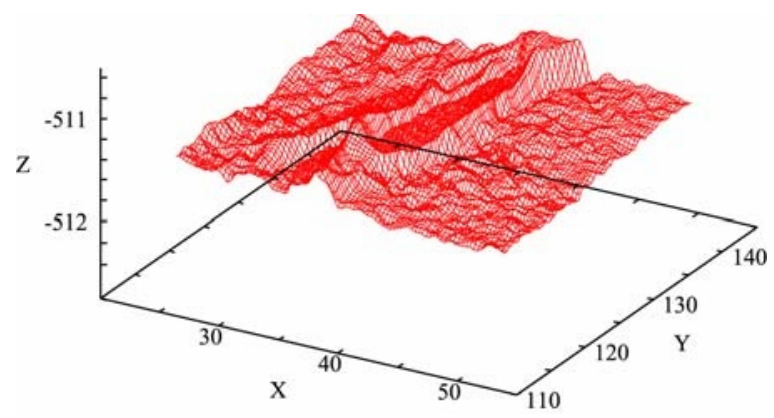

Fig. 3. Surface shape by regular meshes for a small area of an oil painting
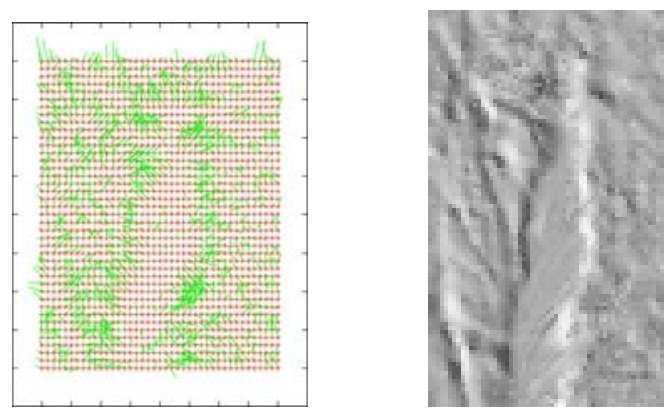

Fig. 4. Array of surface normals by a needle map (left) and by an image (right)

\subsection{Reflection Model}

Let us suppose an oil painting. The surface material of this object consists of a thick oil layer. Sometimes the surface is covered with vanish for the protection or decoration of the painting surface. Therefore, gloss and highlight appear on the surface. This type of object is regarded as an inhomogeneous dielectric material. Light reflected from the object surface is decomposed into two additive components, the specular reflection and the diffuse reflection.

The Torrance- Sparrow model [6] is used for describing the reflection properties of an oil painting. Then the spectral radiance $Y(\lambda)$ from a surface is expressed as

$$
Y(\lambda)=(\mathbf{N} \cdot \mathbf{L}) S(\lambda) E(\lambda)+\beta \frac{D F G}{\mathbf{N} \cdot \mathbf{V}} E(\lambda),
$$

where the first and second terms represent, respectively, the diffuse and specular components. Vectors $\mathbf{N}, \mathbf{V}$, and $\mathbf{L}$ represent the global surface normal vector, the view vector, and the illumination directional vector. $S(\lambda)$ is the surface-spectral 
reflectance, and $E(\lambda)$ is the illuminant spectrum. The specular component in Eq.(1) consists of three terms: $D$ is a function providing the index of surface roughness defined as $\exp \left\{-\ln (2) \varphi^{2} / \gamma^{2}\right\}$, where $\varphi$ is the angle between the normal vector $\mathbf{N}$ and the bisector vector of $\mathbf{L}$ and $\mathbf{V} . \quad G$ is a geometrical attenuation factor. $F$ represents the Fresnel reflectance. $\beta$ represents the intensity of the specular component.

Therefore, $\mathbf{N}, S(\lambda), \gamma$, and $\beta$ are the unknown parameters to be estimated.

\section{Estimation of Surface Reflection}

\subsection{Pixel Classification}

The observed image of an oil painting by the multi-band imaging system is decomposed into two additive components of the diffuse reflection and the specular reflection, as shown in Figure 5. The surface-spectral reflectance function is estimated from the diffuse reflection component. The reflection parameters are estimated from the specular reflection component. We have devised a procedure to detect the diffuse reflection component at each pixel point of the multiple images acquired under different illumination directions.

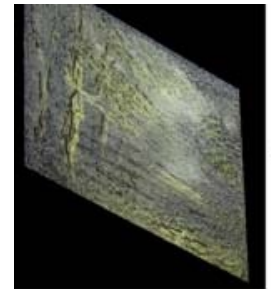

Sensor outputs

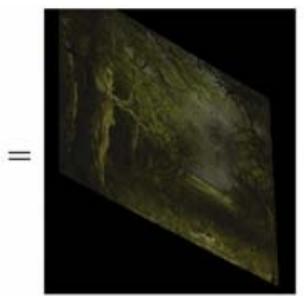

Diffuse component

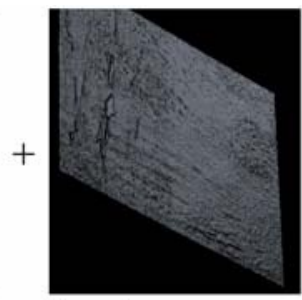

Specular component

Fig. 5. Decomposition of the Observed image into reflection components

\subsection{Spectral Reflectance Estimation}

The sensor outputs for a diffuse object are described as

$$
\rho_{k}=\int_{400}^{700} S(\lambda) E(\lambda) R_{k}(\lambda) d \lambda+n_{k},(\mathrm{k}=1,2, \ldots, 6),
$$

where $R_{k}(\lambda)$ is the spectral sensitivity of the $k$-th sensor, and $n_{k}$ is noise. Let $\boldsymbol{\rho}$ be a six-dimensional column vector representing the camera outputs, and $\mathbf{s}$ be a $n$ dimensional vector representing the spectral reflectance $S(\lambda)$. Moreover, define a 6xn matrix $\mathbf{H}\left(\equiv\left[h_{k i}\right]\right)$ with the element $h_{k i}=E\left(\lambda_{i}\right) R_{k}\left(\lambda_{i}\right) \Delta \lambda$.Then the above imaging relationships are summarized in the matrix form

$$
\boldsymbol{\rho}=\mathbf{H ~ s}+\mathbf{n} .
$$


When the signal component $\mathbf{s}$ and the noise component $\mathbf{n}$ are uncorrelated, the Wiener estimator gives an optimal solution as

$$
\hat{\mathbf{s}}=\mathbf{R}_{s s} \mathbf{H}^{\mathrm{t}}\left[\mathbf{H} \mathbf{R}_{s s} \mathbf{H}^{\mathrm{t}}+\sigma^{2} \mathbf{I}\right]^{-1} \boldsymbol{\rho}
$$

where $\mathbf{R}_{s s}$ is an nxn matrix representing a correlation among surface spectral reflectances. We assume white noise with a correlation matrix $\sigma^{2} \mathbf{I}$.

\subsection{Model Parameter Estimation}

The sensor output with the maximal intensity among the set of outputs under different illumination directions has the possibility of including the specular reflection component. To detect the specular component, we first calculate the output vector $\boldsymbol{\rho}_{\mathrm{D}}$ for the diffuse component by using the estimated reflectance $\hat{S}(\lambda)$. Next, select the maximal sensor output $\boldsymbol{\rho}_{\mathrm{M}}$ among all observations, and define the difference $\boldsymbol{\rho}_{\mathrm{S}}=\boldsymbol{\rho}_{\mathrm{M}}-\boldsymbol{\rho}_{\mathrm{D}}$.

The specular function of the Torrance-Sparrow model is fitted to the specular data extracted from the entire pixel points. Since the specular component at any pixel has the same spectrum as the light source, the parameters are estimated based on the statistical distribution of the specular component. Define the intensity of the specular component as $\left\|\boldsymbol{\rho}_{\mathrm{S}}\right\|$, and normalize the specular component as $\rho_{\mathrm{S}}=\left\|\boldsymbol{\rho}_{\mathrm{S}}\right\|\left(\cos \left(\theta_{i}\right) \cos \left(\theta_{r}\right)\right) /(G F)$. We minimize the sum of the fitting error

$$
e=\sum_{x}\left\{\rho_{\mathrm{S} x}-\beta D\left(\varphi_{x}, \gamma\right)\right\}^{2},
$$

where $\rho_{\mathrm{S} x}$ and $\varphi_{x}$ are the specular intensity and angle at different pixel point $x$. The parameters $\gamma$ and $\beta$ minimizing the error are solved as a solution of the nonlinear fitting problem.

\section{Image Rendering}

\subsection{Prediction of Chromatic Adaptation}

There are many kinds of chromatic-adaptation and color-appearance models (e.g., see [7]-[9]). These models consist of complicated equations with many parameters. Therefore, the previous models are difficult to apply to the problem of image rendering of art paintings in the present study.

We have proposed a color prediction method for incomplete chromatic adaptation that is based on an extended version of the von Kries model using correlated color temperature scale [10]. We use Illuminant D65 as reference and Illuminant A as test. The adaptation process is considered incomplete adaptation along the color temperature scale. Then, an incomplete adaptation index $d(0 \leq d \leq 1)$, representing the 
degree of chromatic adaptation, is introduced on the color temperature scale between the test illuminant A and the reference illuminant D65.

Because the color temperature scale in kelvin $\mathrm{K}$ is not correlated to perceived color differences, we use a reciprocal megakelvin temperature scale. The unit of this scale is the reciprocal megakelvin $\left(\mathrm{MK}^{-1}\right)$, and a given small interval in this scale is approximately perceptible. Let $\left[M K^{-1}\right]_{\mathrm{T}}$ and $\left[M K^{-1}\right]_{\mathrm{R}}$ be the reciprocal temperatures of the test illuminant and the reference illuminant, respectively. Then, the color temperature of adaptation illumination corresponding to the index $d$ is determined as

$$
T_{\mathrm{D}}=10^{6} /\left(\left(\left[M K^{-1}\right]_{\mathrm{R}}-\left[M K^{-1}\right]_{\mathrm{T}}\right) d+\left[M K^{-1}\right]_{\mathrm{T}}\right) .
$$

A proper value of the adaptation index $d$ is determined on matching experiments between real paintings under Illuminant $\mathrm{A}$ and the images on the monitor.

\subsection{Rendering Algorithm}

Images of the target paintings are created using the estimated surface normals and spectral reflectances at all pixel points, and the above determined reflection model. A ray-casting algorithm is adopted for the image rendering under parallel rays from the light source.

For the purpose of accurate color image rendering, the color images of art paintings are not represented by RGB values, but represented by the CIE tristimulus values $\mathrm{XYZ}$. We calculate the XYZ values at each pixel by using the spectral radiance $Y(\lambda)$ and the CIE standard color-matching functions.

Therefore, the image of an art painting under a specified illuminant condition is represented as an array of the tristimulus values XYZ. The color values of each pixel are then transformed by taking the chromatic adaptation effect into account. A computational procedure for this color transformation is given in Ref. [10]. It uses a von Kries-type transformation. The entire transformation process is summarized as

$$
\left[\begin{array}{c}
X_{\mathrm{p}}(D) \\
Y_{\mathrm{p}}(D) \\
Z_{\mathrm{p}}(D)
\end{array}\right]=\mathbf{M}^{-1} \mathbf{W} \mathbf{M}\left[\begin{array}{c}
X \\
Y \\
Z
\end{array}\right],
$$

where $\mathbf{M}$ is a $3 \times 3$ transformation matrix into the cone responses, and $\mathbf{W}$ is a diagonal matrix with the elements of gain coefficients determined by the incomplete adaptation index $d$.

\section{Application Results}

Figure 6 shows the oil painting of "Flowers." The surface of this painting is covered with a transparent oil vanish. This object was measured with the six spectral channels in the image size of 1070x 1503 pixels. The surface normals at all pixel points were estimated using the photometric stereo method to the image intensity data by the diffuse reflection. Figure 7 shows an image rendering the estimated surface normals for 
the rectangular area of "Flowers" in Figure 6. We can see big roughness at the petals.These results are much more precise than the measurement by the laser range finder.

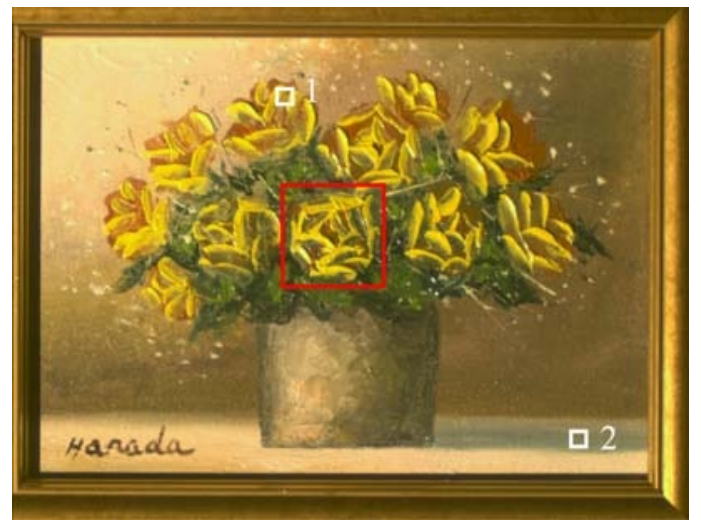

Fig. 6. Oil painting "Flowers"

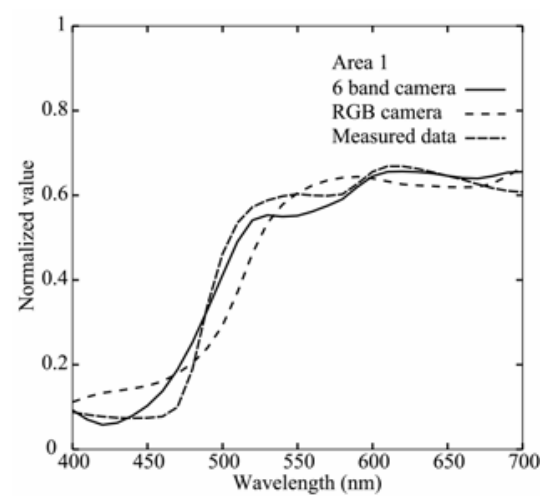

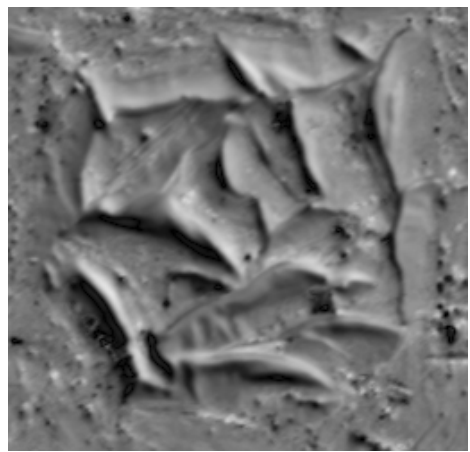

Fig. 7. Images of the estimated surface normals

Fig. 8. Estimation results of surface-spectral reflectances for Area 1 and Area 2

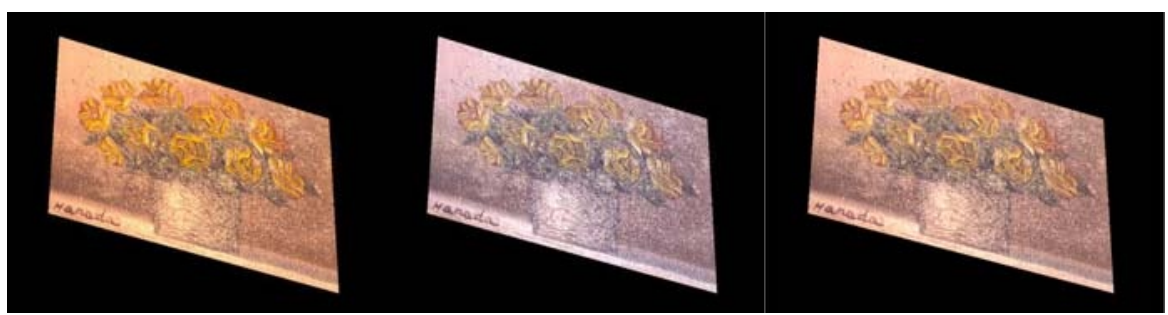

Fig. 9. Image rendering results. (Left: colorimetric rendering under an incandescent illumination, Middle: image rendered under a fluorescent light source of D65, Right: Image rendered with the incomplete chromatic adaptation effect under an incandescent illumination) 
Next, the surface spectral reflectances were estimated at all pixel points. Figure 8 shows the estimation results for Area 1 and Area 2 shown by white rectangles in Figure 6, where the bold curve, the dashed curve, and the broken curve represent, respectively, the estimates by the present method, the direct measurements, and the estimates by the previous method using an RGB camera with three spectral channels. We can see that the present method using the multi-spectral imaging system recovers the detailed shape of spectral reflectance.

We have executed the visual experiment for predicting incomplete chromatic adaptation to several oil paintings. This experiment was conducted by using the method of memory matching between the target painting under a real Illuminant A and the images on a calibrated monitor. The most proper prediction was performed with the index value of about $d=0.5$.

All the estimates of the surface properties were combined for creating computer graphics images of the oil painting under different illumination and viewing conditions. Figure 9 shows the image rendering results. The left picture represents the image under an incandescent light source that is based on the colorimetric rendering without any chromatic adaptation effect. The middle picture represents the image under a fluorescent light source with the color temperature of D65. The right picture represents the image created with the incomplete chromatic adaptation effect.

\section{Conclusion}

The present paper has described an approach to digital archives of art paintings by considering the surface properties and the perceptual effect. A multi-band imaging system with six spectral channels was used for observing the painting surfaces. We have presented algorithms for estimating the surface properties from the image data, which includes surface normals, surface spectral reflectance, and reflection model parameters. All these estimates were combined for rendering realistic images of the original painting under a variety of illumination and viewing conditions.

We have described a chromatic adaptation transform for predicting appearance of the painting under incandescent illumination and producing the full color image on a display device. The incomplete adaptation index representing the degree of chromatic adaptation was defined on the color temperature scale. The von Kries' framework was extended to the algorithm of incomplete chromatic adaptation.

This paper has shown the feasibility of the proposed method for oil paintings. As discussed in 3.2, the surface of a water painting is rough and reflects no specular component that is essentially different from any oil painting. We have found that the surface of a water painting is not Lambertian. The digital archiving problem for water paintings is left for a future work.

\section{References}

[1] K. Martinez et al.: Ten years of art imaging research, Proceedings of the IEEE, vol. 90, No. 1, 2002.

[2] S. Tominaga and N. Tanaka: 3D Recording and Rendering of Art Paintings, Proc. Ninth Color Imaging Conf., pp.337-341, 2001. 
[3] N. Tanaka and S. Tominaga: Measuring and Rendering Art Paintings Using an RGB Camera, Proc. of EUROGRAPHICS, pp.299-306, 2002.

[4] H. Maitre et al.: Spectrophotometric image analysis of fine art paintings, Proc. Fourth Color Imaging Conf., pp.50-53, 1996.

[5] Y. Miyake, et al.: Development of multiband color imaging systems for recording of art paintings, Proc. SPIE: Color Imaging, Vol.3648, pp.218-225, 1999.

[6] K.E.Torrance and E.M.Sparrow: Theory for off-specular reflection from roughened surfaces, J. of Optical Society of America A, Vol.57, No.9, pp.1105-1114, 1967.

[7] Y. Nayatani: A Simple Estimation Method for Effective Adaptation Coefficient, Color Res. Appl. Vol.22, pp.259-268, 1997.

[8] M. D. Fairchild : Color Appearance Models, Addison-Wesley, 1998.

[9] N. Moroney, M. D. Fairchild, R. W. G. Hunt, C. Li, M. R. Luo and T. Newman: The CIECAM02 Color Appearance Model, Proc. Tenth Color Imaging Conf., pp.23-27, 2002.

[10] Tominaga, M. Nakagawa, and N. Tanaka: Image Rendering of Art Paintings, Proc. Twelfth Color Imaging Conf., pp.70-75, 2004 